\title{
The Role of SmallSats in Scientific Exploration and Commercialization of Space
}

\author{
Azita Valinia ${ }^{1}$, Joe Burt, Thai Pham, and Opher Ganel \\ Astrophysics Flight Projects Division, NASA Goddard Space Flight Center \\ 8800 Greenbelt Rd, Greenbelt, MD USA, 20771
}

\begin{abstract}
Over the past decade, SmallSats have been established as having great potential for science exploration and commercialization of space. The SmallSat revolution aims to decrease the cost of space development, making space exploration accessible to students, educators, and public citizens. These efforts have focused on miniaturization of instruments and space platforms, as well as reducing their cost, mass, and needed power. In addition to enabling scientific exploration, SmallSats provide affordable means for the public to purchase remote sensing and communication products on a global scale.
\end{abstract}

SmallSat mission concepts are particularly powerful when they are deployed in distributed architecture or constellations. For example, the most promising observation techniques for global science measurements of the Earth system and space weather require multi-point distributed observations of the Earth system at a feasible cost. The high cost of access to space has long been a barrier, especially with the prohibitive cost of large satellites. Affordable SmallSat constellations can be game-changers, enabling scientific exploration as well as commercial global data products.

In this paper, we highlight investments made by NASA to date (specifically a study in developing and prototyping a SmallSat platform with standard interfaces), along with several example mission concept scenarios in Earth and space science (astrophysics, heliophysics, and planetary) applications that can be achieved using this platform.

Keywords: NASA, smallsats, astrophysics, heliophysics, Earth science, technology development, optics, telescope, detector, laser, CapSat, CapSIT

\section{INTRODUCTION}

Over the past decade, a movement has been underway to decrease the cost of space development, making space accessible to all sectors of society. Space agencies, as well as the commercial and defense sectors, are considering innovative uses of disaggregated SmallSat constellations to accomplish at lower cost and more rapid turnaround the same objectives previously achievable only via large singular spacecrafts, with their long lead times and high cost. A natural consequence of these efforts (we call it collectively the "SmallSat revolution") has been to lower the cost of access to space, since smaller satellites can be launched more affordably via a "rideshare" scheme launching with larger primary payloads. Not only are SmallSats having high impact on science and space exploration, they also provide an affordable means for the public to purchase remote sensing and communication products on a global scale.

In general, SmallSats represent an emerging class of satellites, with their small size, mass, and cost allowing us to do more with less. Specialized needed technology developments for deployment on SmallSats include miniaturization (size and mass), communications, propulsion, power, guidance, navigation and control, instrumentation, bus subsystems, and advanced manufacturing techniques. Potential advances could lead to development of miniature radio and navigation devices, low-power laser communications, radiation-tolerant computers, energy storage devices, and electric propulsion for deep space missions.

In 2016, the NASA Earth Science Technology Office (ESTO), funded a study to develop a prototype modular SmallSat with standard interfaces. The concept, known as Capsulation Satellite (CapSat), is a low-cost, 3-axisstabilized (predicted pointing performance better than a few arcsec using two star trackers), modular, standardized spacecraft, with a pressurizable volume allowing ruggedized commercial-off-the-shelf (COTS) hardware to be flown reliably in space at a cost (per $\mathrm{kg}$ ) $20 \times$ cheaper than the average CubeSat.

In this paper, we discuss the CapSat architecture and its associated CapSat Science Instrument Tube (CapSIT). We describe several scenarios in Earth science, planetary science, heliophysics, astrophysics, and space operations; illustrating how CapSats can be used for scientific exploration, space situational awareness, data commercialization, and technology demonstration and maturation. We also discuss future potential NASA opportunities whereby principal investigators could take advantage of this novel and innovative concept to fly payloads on this platform at a small fraction of typical bus and launch costs.

\footnotetext{
1*Azita.Valinia@nasa.gov; phone: +1 301-286-5039
} 


\section{CAPSULATION SATELLITE ARCHITECURE}

CapSat [1] is a modularized, pressurizable, thermally controlled spacecraft designed to host ruggedized COTS instrumentation in a terrestrial-like environment on orbit. CapSat takes advantage of a pressurizable volume for both spacecraft and payload systems. It builds on NASA's tradition of Hitchhiker Get Away Special (GAS) Cans that flew on the Space Shuttle. Hitchhiker flew over 200 successful missions over two decades (1984-2003), managed out of NASA Goddard Space Flight Center (GSFC).

The pressurizable volume provides multiple benefits, primarily in thermal design. By maintaining one atmosphere of pressure inside the CapSat, materials that might otherwise outgas, and/or fail, and/or cause significant contamination issues, are no longer a concern. This also means that certain vibration-absorbing materials/designs used in COTS hardware can be used on orbit. Additionally, printed circuit boards do not have to be redesigned for thermal requirements (i.e. moving hot chips to the cold side, etc.), plus conformal coating and contamination bake-outs are no longer required.

CapSat was specifically designed to take advantage of the United States Air Force (USAF) Rideshare Program and the Evolved Expendable Launch Vehicle Secondary Payload Adaptor (ESPA) ring. The ESPA ring comes in two sizes: standard and Grande. CapSat has two main configurations, one designed for the ESPA Grande, and the other for the standard ESPA ring. The ESPA Grande CapSat version is a hockey-puck-shaped spacecraft bus measuring approximately 40 inches in diameter and 20 inches in height. This version takes full advantage of the ESPA Grande's 300-kilogram capability per attachment point (with 4/5 attachment points per ring). This CapSat configuration can make available to the payload over $100 \mathrm{~kg}$ and $100 \mathrm{~W}$ (Fig. 1). The second CapSat configuration is designed around a pair of CapSITs (Fig. 2).
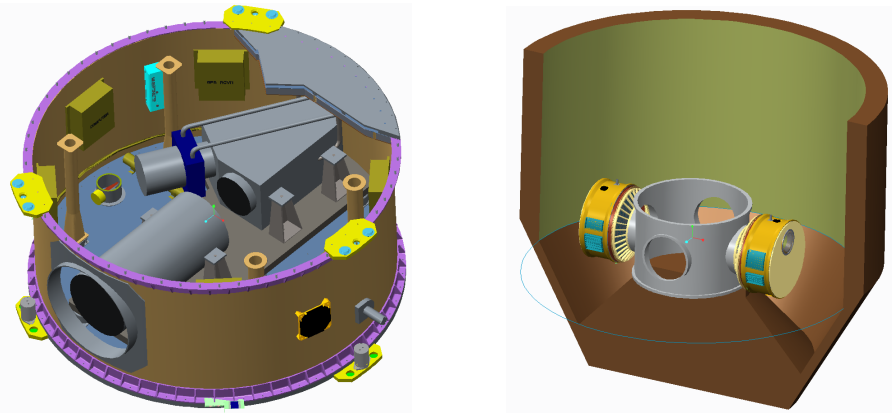

Fig. 1. ESPA-Grande-based CapSat bus architecture (left) and two CapSats mounted on an ESPA Grande (right).
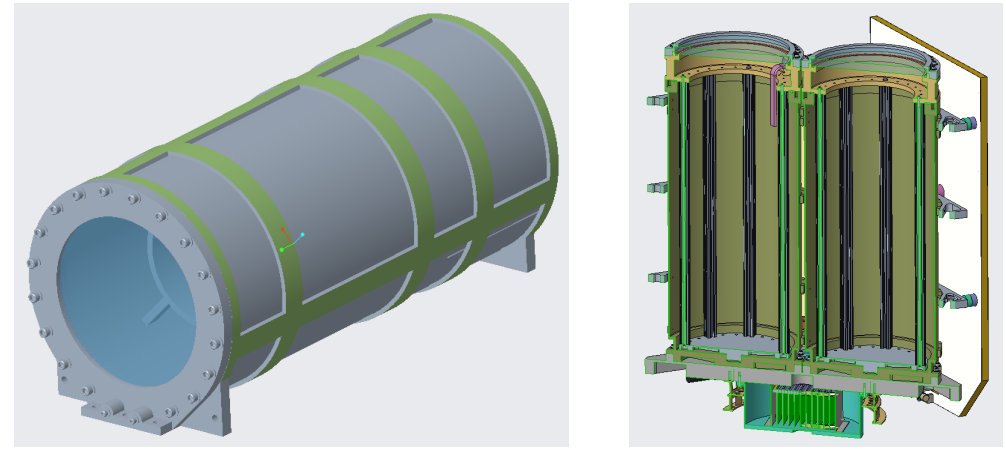

Fig. 2. Capsulation Satellite Science Instrument Tube (CapSIT, left) and cutaway view (right).

The standard-ESPA-based CapSat (Fig. 3) has two 0.78-square-meter solar arrays capable of producing $138 \mathrm{~W}$ each, for a total of $276 \mathrm{~W}$ and a LEO orbit average power of $135 \mathrm{~W}$. CapSat also has a 1-meter high-gain antenna (HGA) capable of X-band downlink rates in excess of $400 \mathrm{Mbps}$. CapSat flight software uses NASA's open-source core Flight Executive (cFE), developed with decades of GSFC heritage, and now approved for Class-A human-rated use. A version of Microsoft Windows was successfully run on a CapSat command and data handling (C\&DH) system within the core Flight System (cFS), demonstrating plug-and-play instrument software integration for a new type of Landsat Thermal Imaging Sensor in 2016 (see Section 4). CapSat ground systems use the Integrated Test \& Operations System (ITOS), developed at GSFC in 1990 and available as a commercial product since 2000. ITOS has flown on missions such as Landsat, LRO, DSCOVR, Fermi (GLAST), LADEE, NuSTAR, ICESAT-2, and is planned to fly on the upcoming ICON Explorer mission. ITOS supports instrument and spacecraft development as well as full-observatory on-orbit operations. 



Fig. 3. Left to right: Standard-ESPA-based CapSat bus architecture; Top view showing two CapSITs; Bottom view showing separate pressurized volume holding spacecraft electronics; and six CapSats mounted on a standard ESPA.

\section{STANDARDIZING THE INSTRUMENT INTERFACES}

On the payload side, CapSat's pressurizable volume provides a plug-and-play-type interface for lab instruments so that they can be readily flown on orbit. The CapSIT (Fig. 2) is a standardized interface allowing independent development of multiple instruments that can be integrated quickly into the bus. CapSIT is also fully qualified for flight independent of the CapSat spacecraft and can be fully pressurized (or not) as needed. Each CapSIT is $\sim 1 \mathrm{ft}$ in diameter and $2.5 \mathrm{ft}$ in length, providing an instrument volume equal to approximately $53 \mathrm{U}$ per tube, can contain up to $17.5 \mathrm{~kg}$ and accommodate as much as $100 \mathrm{~W}$. A pair of CapSITs double the above numbers to $106 \mathrm{U}, 35 \mathrm{~kg}$, and $200 \mathrm{~W}$. The CapSIT is designed with standard interfaces for rapid interchangeability. To support this interchangeability without affecting coupled loads, lower-mass payloads are "topped off" with dummy masses up to the full mass allowance. Tubes can be delivered to an instrument developer in a kit form, including software to allow testing through commercial standard data interfaces. The mechanical ICD specifies CG location and minimum frequency responses. The electrical ICD includes EMI/EMC and in-rush current requirements. Tubes can be flown pressurized, unpressurized, or partially pressurized. Because of form-factor interchangeability, qualified tubes can be delivered or swapped out very late in the integration and test (I\&T) process. Finally, tubes can be flown pressurized, unpressurized, or partially pressurized. This means that instrument development can be decoupled from spacecraft build schedules, with the only requirement that CapSIT-to-spacecraft I\&T has to occur far enough in advance of launch to meet rideshare requirements. Given the standard interfaces, the I\&T process is far shorter than with nonstandard buses and with payloads not built to standard volume, mass, CG location, and interfaces.

\section{CAPSAT POTENTIAL MISSION CONCEPT SCENARIOS}

CapSat is capable of observing in all directions (Fig. 4): Earth-pointing, sun-pointing, and stellar-pointing. As such, it can be used for a wide range of science and other applications: Earth science, planetary science, heliophysics, astrophysics, dynamic studies, etc. CapSat excels in its ability to make measurements using low-cost terrestrial COTS hardware using pressurized technology. For example, flying a COTS 10-inch-diameter telescope in a thermally stable, pressurized volume yields an optically stable viewing capability at very low cost. One can envision taking an instrument directly out of the lab, ruggedizing it, and flying it with minimal delay.

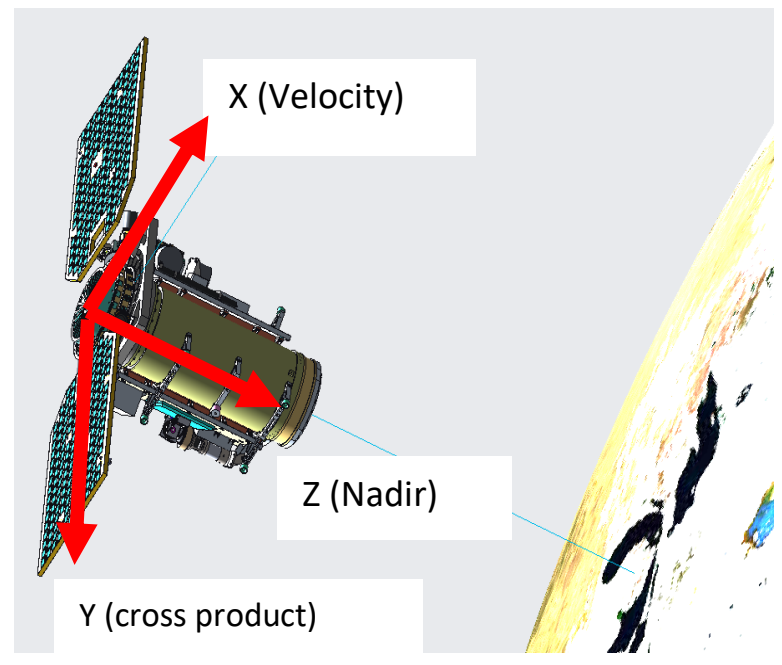

Fig. 4. CapSat can point in all directions enabling a variety of scientific exploration and commercial opportunities. 


\section{Earth Science Scenarios}

Several mission-concept architectures can be envisioned using a constellation of CapSats to provide an array of Earth-science parameter data for commercial utilization. ESTO funded the CapSat study specifically focusing on an Earth-science application. An advanced photodetector camera for land imaging, the Strained-Layer Superlattice (SLS) IR Detector Camera, with flight heritage from LandSat 8, was considered as a potential payload on a mission architecture employing a constellation of CapSats. SLS is the next generation of a previous IR camera system based on Quantum-Well Infrared Photodetector (QWIP) technology. SLS was chosen for the camera because it was already advanced in technology readiness level (TRL) [2] and was a good fit for the CapSat mission. Compared with its QWIP predecessor, the SLS detector is over 10 times as sensitive, can operate at warmer temperatures, and has a wider range of spectral selectivity. It is also relatively inexpensive to build, making it the ideal candidate for outfitting a constellation of CapSats for land imaging purposes. The distributed mission architecture design can be tailored to provide low-cost land data for commercial purposes.

\section{Astrophysics Scenarios}

A variety of science scenarios could benefit from a CapSat application. These include a more capable version of HaloSat [3], a $6 \mathrm{U}$ CubeSat designed to measure X-ray signals from oxygen atoms surrounding the Milky Way galaxy to establish if hot galactic halos can explain part or all of the so-called missing baryon problem. Next, a mission using available lobster-eye X-ray optics in $10 \times 10 \times 60 \mathrm{~cm}^{3}$ packages to study kilonovas from mergers of compact objects such as binary neutron stars or a neutron star and a black hole. Since the Earth's atmosphere blocks IR and severely attenuates UV light, a single CapSat, or preferably a constellation of CapSats could be very useful to follow up on gravitational-wave signals detected by LIGO. This would generate a multi-messenger capability by adding rapid observation of transient UV and IR signals arriving from the same part of the sky. Exoplanet spectroscopy could also benefit from one or more CapSats.

\section{Heliophysics Scenarios}

Taking advantage of how well-suited CapSat is for imaging missions, one could develop mission concepts such as a combined EUV/coronagraph imaging of the sun and its surroundings, space-based UV and optical imaging of auroras, or studies of terrestrial gamma-ray flashes (TGFs) likely generated by major thunderstorms (similar to the Firefly 3U CubeSat mission [4]).

\section{Planetary Science Scenarios}

A wide variety of planetary science scenarios might be able to benefit from a CapSat-like capability, especially given the new NASA Planetary Division program, Small Innovative Missions for Planetary Exploration (SIMPLEx). Such scenarios include fly-bys or orbit (and possibly crash onto) asteroids or comets; fly-bys of Jovian moons such as Europa; deployment to the future Lunar Gateway and from there to the moon. Such missions might carry IR imagers to study asteroids and comets; mass spectrometers to measure the density and species of molecules in cometary tails, European plumes, etc.; multi-band instruments to study the formation of water on the moon and elsewhere, possibly resulting from interactions of solar wind and/or galactic cosmic-ray protons with regolith oxygen atoms. Another concept could be preparing a large number of IR-detector-instrumented CapSats, and launching them on rideshare opportunities whenever a near-Earth object approaches and we have sufficient advance notice.

\section{Other Space-Based Applications}

Under a NASA Science Mission Directorate (SMD) study for FY-2020, the CapSat team will begin developing the Debris Resistive Acoustic Grid Orbital NASA-Navy Sensor (CapSat-DRAGONS), to make in-situ orbital debris measurements. Several NASA Earth-science spacecraft, threatened by orbital debris, are flying in the so-called A-Train orbit between 700-1000 kilometers above Earth. This is where NASA is considering deploying new sensors via CapSat for a quick and inexpensive space situational awareness mission. The mission's direct measurements would be used to update current debris models. This, in turn, would provide more accurate risk assessments enabling costeffective shielding designs for current and future NASA space missions.

While a pressurized volume is not required for this type of sensor, CapSat provides a pressurized volume for sensor electronics and processor boards. This maximizes the options for using lower-cost COTS devices while providing an enhanced, thermally stable on-orbit environment, promoting longevity. In fact, inside the pressurized tube, temperatures may be more stable than the diurnal temperature cycle in a lab. This obviates the need for thermal-vac testing of those electronics, dramatically reducing I\&T cost and schedule.

A variety of other applications could also be built around the CapSat capabilities and the rideshare program. These include e.g. electron density profiling survey using radio occultation of GPS signals at the Earth's limb. Another 
application could mount hyperspectral detectors in an Earth-observing CapSat. A third could use a single CapSat or a constellation of CapSats to measure orbital debris sizes, velocities, and trajectories and create a robust orbital debris model. This could be done by releasing the CapSats at high LEO altitudes (e.g., $800 \mathrm{~km}$ ) where they would remain for several weeks. Then, the satellites would use onboard propulsion to gradually descend in multiple steps, taking measurements down to $\sim 300 \mathrm{~km}$. A fourth scenario would mount sensitive accelerometers in one or a constellation of CapSats, and use those to measure neutral particle density at various altitudes. Finally, a single CapSat or a constellation of CapSats could bring to life an idea of providing laser guide stars for ground-based adaptive-optics imaging of GEO satellites [5]. This application too could benefit from an onboard propulsion system.

\section{CAPSAT AFFORDABILITY}

There are several reasons the mission concept scenarios described above (or others that can similarly be envisioned) are far more affordable as CapSat payloads than current space missions (per kg). First, the pressurized environment of either CapSat or CapSIT, minimizes needed technology development, space flight validation, and testing. Researchers can take an instrument from the lab, ruggedize it, and shortly thereafter, fly it - "If it fits (in the architecture), ifflies!"

Second, standard interfaces and a plug-and-play approach enable quick spacecraft and launch vehicle (via the ESPA ring) I\&T. This eliminates the need for customized designs, minimizing cost and longer schedule.

Finally, CapSat takes advantage of unused launch-vehicle mass-to-orbit capability via the USAF Rideshare program and planned NASA rideshare opportunities, being specifically designed to mate to an ESPA ring. Almost all future NASA missions will be providing rideshare opportunities. This capacity typically goes unused. Cost per kg of space missions tends to be high for large and small missions (that's why typical CubeSat cost-to-launch is nearly $\$ 1 \mathrm{M} / \mathrm{kg}$ ). However, there is a sweet spot in the middle, allowing CapSat to place 180-320 kg of on-orbit mass for $<1 / 20$ of the cost of CubeSat on-orbit mass.

\section{CHALLENGES AND OPPORTUNITIES}

Between November 2020 and the end of 2026 (just over 6 years), NASA is expected to launch at least 16 missions with a total excess capacity that can accommodate 81 rideshare spacecraft with a total mass in excess of 33 metric tons, attaching to ESPA or ESPA Grande rings. Orbits include polar circular, polar sun-synchronous, L1, and Earth escape (one to the Trojan asteroids and one to rendezvous with the metallic asteroid, 16 Psyche). It is estimated that USAF and industry may launch at least as many missions during that same period carrying ESPA-ring-based rideshare secondary payloads. SmallSats such as CapSat provide an extremely low-cost means to use this excess mass-to-orbit capacity.

While the Hitchhiker GAS Cans allowed very efficient use of excess mass- and volume-to-orbit on the Space Shuttle, other attempts at standard buses that would allow lower-cost accommodation for a large set of missions (e.g., the intent to use the Multi-Mission Spacecraft design for EUVE and XTE) ended up tailoring their initial spacecraft to such an extent that cost savings evaporated. This points to a significant challenge facing a CapSat or other standard SmallSat bus concept - the understandable desire by scientists to maximize the science return of their specific mission, which usually requires a custom spacecraft.

To avoid this, an eventual CapSat program has to find customers willing to accept an as-built design as good enough given its low cost. Many payload-unique requirements can be met within the payload's technical and budgetary resources, e.g. using low-cost COTS SmallSat components accommodated within the CapSITs. This will avoid increasing bus costs for all missions to meet the requirements of each specific payload. We have to accept that while CapSat is a great fit for many potential applications, some will need to use larger (more expensive) standard options, including e.g. propulsive ESPA rings, AQUILA, etc., beyond the scope of this article, while others will opt for one-off customized buses. Those applications wishing to take advantage of CapSat will need to design their payload to the standard, as-built bus capabilities, rather than expecting a tailored bus designed to meet their payload-specific requirements. This is similar to the difference between a bare-bones low-cost fare such as on Spirit Airlines, as compared to the far more expensive, but all-inclusive option of flying first-class on a traditional airline.

Another challenge has to do with the institutional mindsets that have made NASA very successful with payloads flying on large one-off spacecraft. The much lower mission costs enabled by CapSat allow a very different risk posture than even a Class D mission. However, this requires adopting an agile mindset closer to that of suborbital rocket and CubeSat missions, rather than imposing the same safety and mission assurance (SMA) requirements as on higher-cost missions.

Finally, mission assessment tools such as the Technical, Management, Cost, and Other (TMCO) process use parameterized cost models based on historic as-flown costs of past missions. The expected cost of a build-to-print CapSat is much lower than any other bus of similar mass, volume, and power. Thus, unless they are updated 
appropriately, parametric cost models will greatly over-estimate mission lifetime cost for any mission based on a CapSat bus. In this new paradigm, with an inexpensive bus accommodated via rideshares, mass-to-orbit is no longer a significant mission-cost driver. Similarly, given the estimate of six or more annual rideshare opportunities at minimal accommodation cost, rideshares should now be seen as a risk mitigation, rather than a schedule risk. If either the primary or rideshare payload is delayed, the standard mass, CG location, and ESPA interface allow a rideshare mission to hop onto another launch vehicle within a few months of when they are ready.

\section{SUMMARY AND LOOKING TO THE FUTURE}

We described the CapSat architecture, along with many case scenarios where the platform can be used for broad science exploration from space as well as commercial and other applications. Three main advantages of this architecture are: 1) short payload development times using COTS hardware and minimally re-designed and ruggedized lab instruments; 2) standard interfaces; and 3) rideshare opportunities on future NASA and other space flight missions. All three factors render total mission costs far more affordable than any comparable concepts in this class.

These mission scenarios can be enabled via government, industry, and academic partnerships involving NASA or other government agencies. For example, future scientific CapSat mission concepts can be enabled via successful proposals to various NASA announcement of opportunities (AO), such as the astrophysics and heliophysics Explorers Missions of Opportunity (MOO) and the Earth Venture (EV) AOs. Several CapSat mission architectures can be enabled via industry and government partnerships that can provide Earth science related data to the public at low cost.

Several factors, including cost effectiveness, are driving NASA toward smaller missions and platforms, creating new opportunities such as the Planetary Division's SIMPLEx program affording up to $\$ 55 \mathrm{M}$ per mission, as well as a recent Astrophysics Division study of possible new \$35M-class missions, and its $\$ 5 \mathrm{M} /$ year CubeSat investment. In addition, the government is pushing for greater industry involvement in space missions. Whichever organizations are able to adopt the mindset required to take the greatest advantage of SmallSat opportunities, and who are willing to use low-cost standard buses, will reap the greatest benefits from the "SmallSat revolution."

\section{REFERENCES}

[1] J. Burt and D. Steinfeld, “Capsulation satellite or CapSat: A low-cost, reliable, rapid-response spacecraft platform," 1-13. 10.1109/AERO.2017.7943937 (2017)

[2] “NASA Systems Engineering Processes and Requirements (NPR 7123.1B)," Appendix E (Technology Readiness Level) available at https://nodis3.gsfc.nasa.gov/displayDir.cfm?Internal ID=N_PR 7123 001B \&page name=AppendixE

[3] B. Dingwall, et al., "HaloSat - A CubeSat to study the Hot Galactic Halo", The Committee on Achieving Science Goals with CubeSats (2015)

[4] J. Klenzing et al., "FIREFLY: A CubeSat Mission to Study Terrestrial Gamma-ray Flashes”, AGU Fall Meeting Abstracts (2009)

[5] W.A. Marlow et al., "Laser-Guide-Star Satellite for Ground-Based Adaptive Optics Imaging of Geosynchronous Satellites," Journal of Spacecraft and Rockets, 54(3), DOI:10.2514/1.A33680 (May-June 2017) 